\title{
A DELAYED NONLINEAR PBPK MODEL FOR GENISTEIN DOSIMETRY IN RATS *
}

\author{
MICHAEL G. ZAGER ${ }^{\dagger}$, HIEN T. TRAN ${ }^{\ddagger}$, AND PAUL M. SCHLOSSER $\S$
}

\begin{abstract}
Genistein is an endocrine-active compound found in soy products. It has been linked to beneficial effects such as mammary tumor growth suppression and adverse endocrine-related effects such as reduced birth weight in rats and humans. In its conjugated form, genistein is excreted in the bile, which is a significant factor in its pharmacokinetics. Experimental data suggest that genistein induces a concentration-dependent suppression of biliary excretion. In this article we describe a physiologically based pharmacokinetic (PBPK) model that focuses on biliary excretion with the goal of accurately simulating the observed suppression. The mathematical model is a system of nonlinear differential equations with state-dependent delay to describe biliary excretion. The model was analyzed to examine local existence and uniqueness of a solution to the equations. Furthermore, unknown parameters were estimated, and the mathematical model was compared against published experimental data.
\end{abstract}

Key words. genistein, dosimetry, pharmacokinetics, state-dependent delay, endocrine-active compound

1. Introduction. The presence of endocrine-active compounds (EAC) in the environment has become a concern to environmental biologists and toxicologists. Researchers have suggested that numerous health effects, both adverse and beneficial, may be caused by exposures to these compounds. The suggestions stem from observations that include field studies on wildlife and human cultures $[1,2,3]$, controlled experiments on laboratory animals and human volunteers [4], and critical literature reviews [32]. Phytoestrogens, a class of EAC that are naturally plant-derived, bind to the estrogen receptor as either agonists or antagonists [5]. Because these chemicals are plant-derived, exposure to them is primarily through the diet. In particular, the phytoestrogen genistein is a significant component of these dietary exposures [6], including soybean-derived foods with high genistein concentrations, such as tofu and soy-derived infant formula [7]. Genistein is believed to have a variety of effects ranging from health benefits like cancer prevention $[4,8,9,10]$ to adverse effects such as endocrine disruption and promotion of carcinogenic tumor growth [11, 12, 29]. Consequently, the health effects of genistein have been extensively studied. Endocrine- and cancer-related effects have been found in studies of soy-based infant formula [30,33]. The United States Environmental Protection Agency developed a pilot study using genistein, among other EAC, as part of a proposed multimillion dollar Endocrine Disruption Screening Program designed to screen thousands of EAC and their health effects [22]. Epidemiological studies show that Asian cultures with diets rich in soy products have a lower incidence of breast cancer than western cultures [13, 14, 15]. Experimental studies have shown that treatment with genistein suppresses mammary tumor growth in rats and humans [16, 17]. Experimental prostate cancer treatments using genistein are currently underway in the United States [18]. Other studies have

${ }^{*}$ This research was supported by the American Chemistry Council (formerly the Chemical Manufacturers Association, CMA Agreement Reference Number 9121).

$\dagger^{\dagger}$ orth Carolina State University, Center for Research in Scientific Computation, Box 8205, Harrelson Hall, Raleigh, NC 27695-8205

${ }_{\ddagger}^{\ddagger}$ North Carolina State University, Center for Research in Scientific Computation, Box 8205, Harrelson Hall, Raleigh, NC 27695-8205

${ }^{\S}$ CIIT Centers for Health Research, PO Box 12137, 6 Davis Dr., Research Triangle Park, NC 27709 
shown that genistein produces adverse endocrine-related effects in rats such as low birth weights and reduced anogenital distances [11, 12, 19]. The National Toxicology Program conducted a multigenerational toxicity study on genistein in rats, and various additional studies are currently underway to complement that work. These studies include effects of neonatal treatment of genistein on mice and hormonal effects of dietary administration of genistein on rats $[30,31,33]$.

Physiologically based pharmacokinetic (PBPK) models are often developed to simulate the general dosimetry of a chemical. Due to the apparently intricate nature of the biliary excretion of genistein, PBPK models for the dosimery of genistein have been difficult to develop based on traditional PBPK modeling approaches. The parameters governing the simulation of biliary excretion in the models have been observed to be highly sensitive to the data, although no formal analyses to show the sensitivity have been done to this point. In this article we describe a PBPK model that focuses on biliary excretion with the goal of accurately simulating the observed delay. Development of such a model should inevitably yield more accurate parameters that may be used in general PBPK models for genistein, and will allow for quantification and extrapolation of the relationships between exposure levels and target-tissue dose that occur in the genistein studies. Furthermore, the model can aid in identifying key genistein data gaps, and may be used as a template for developing PBPK models of other compounds that undergo biliary excretion.

Genistein has been shown to undergo enterohepatic circulation [20, 21], where it is excreted via the biliary tract to the gastrointestinal tract and subsequently reabsorbed. Genistein is extensively metabolized via glucuronidation, and most of the genistein excreted in the bile is in its glucuronidated form [20,21]. Once excreted into the intestine, it is quickly cleaved from the conjugated form back into its parent form of pure genistein. We initially developed a preliminary PBPK model for rats that includes the simulation of biliary excretion [26]. In attempting to calibrate the model, we observed that one of the data sets being used strongly suggests a concentrationdependent lag in biliary excretion of genistein [20]. While the ordinary differential equations (ODE) used to describe the model were able to describe the delay observed in the data, this required treating the bile duct as a series of five compartments. We thought that biliary excretion would be more accurately described as a delay process. Therefore state-dependent delay differential equations (DDE) were developed to incorporate the compartments and parameters necessary to simulate biliary excretion under the experimental conditions of the data set described in Sfakianos, et al. [20]. The values of the unknown parameters were estimated by fitting the model to the biliary excretion data.

The organization of the article is as follows. In Section 2, we begin by explaining the Sfakianos data set in detail and its significance. Then, based on those details, we describe the development of the DDE model. In section 3, we prove local existence and uniqueness of a solution to the DDE. In section 4, we explore the inverse problem yielded by the unknown kinetic parameters of the model paired with the experimental data. The methods used in solving this inverse problem are discussed, and the numerical results obtained are presented. In section 5, we establish statistical significance in using a DDE model over a standard ODE model. We discuss the DDE model results in Section 6 and offer future directions in this work that include development of a more biologically descriptive model for simulating genistein biliary excretion. With the exception of Table 1, all tables are found in the appendix directly following section 5 . 
2. Model Development. In the work presented in Sfakianos et al. [20], rats were lightly anesthetized, fitted with bile duct cannulas to collect biliary excretions, and infused in the liver portal vein with one of four different dose rates of ${ }^{14} \mathrm{C}$-labeled genistein dissolved in rat serum for $1 \mathrm{~h}$. Measurements of the rate of excretion of ${ }^{14} \mathrm{C}$ radioactivity were taken every $5 \mathrm{~min}$ from the beginning of the infusion through 65 min (Figure 2.1). Figure 1B is a magnification of the first 20 min into the infusions. Genistein first appears in the bile at the third measurement taken, $10 \mathrm{~min}(0.17$ h) after the beginning of the infusion. At this time point, the lowest infusion rate yields the highest rate of genistein excretion. This trend continues through $15 \mathrm{~min}$ of infusion, and then within 45 min of infusion the highest infusion rate finally yields the highest excretion rate. Thus there is a delay between the start of the infusion and the time when the excretion rate responds proportionately to the dose.

The significance in these data is that Sfakianos [20] reports the only in vivo genistein experiment involving biliary excretion in the literature that includes multiple doses and time intervals in measurement short enough to capture the observed delay. All other published biliary excretion experiments are in time intervals of $20 \mathrm{~min}$ or longer, and no other experiment includes more than one dose. Clearly, this delay would be very difficult to observe with time intervals as large as $20 \mathrm{~min}$, and nearly impossible with a single genistein dose (Figure 2.1). Given the above experimental conditions, we formulated the model as follows. All transport and elimination rates are linear. The transport rates depend on the blood flow rates through the particular tissues of each compartment. The values used for the blood flows were obtained from ILSI [23]. The values for the partition coefficients were obtained from Plowchalk [24]. The volumes of each compartment were calculated from the formula volume $=\frac{\text { mass }}{\text { density }}$ using values for densities and percentages of body mass listed in ILSI [23]. Values and abbreviations used for the volumes are listed in Table 6.2. The elimination rates for the model are currently unavailable in the literature and therefore must be estimated. The abbreviations used for these rates are also given in Table 6.2. The values are listed as "estimated" and are provided in Section 4.

Experimental data suggest that genistein conjugation is saturable [20], that is, the rate at which conjugation occurs increases with the concentration of the compound being conjugated, but at high concentrations the rate asymptotically approaches an upper bound. Therefore Michaelis-Menten kinetics characterized by the parameters $V_{\max }$ and $k_{m}$ are used to describe the rate. However, the values of these parameters are not available in the literature and must be estimated. These parameters are listed in Table 6.2 as "estimated" and the values are provided in Section 4.

In the Sfakianos study, the authors reported that genistein is excreted in the bile exclusively in its glucuronidated form, and they only report data for this metabolite. Although other studies suggest that traces of pure genistein may be found in the bile, they are negligible amounts [21]. So we chose to ignore excretion of unconjugated genistein in the model. Another study has shown that genistein excreted in urine is in its pure and sulfated forms [21]. Hence we have urinary elimination routes for pure and conjugated genistein in our model. We do not distinguish between the glucuronide and sulfate conjugates in the model.

With the above assumptions, the DDE model is split into two submodels; one part tracks pure genistein, and the other tracks conjugated genistein. Our resulting model is depicted in Figure 2.2, and the abbreviations used are defined in Table 6.1. The equations describing the model have the form: 
A

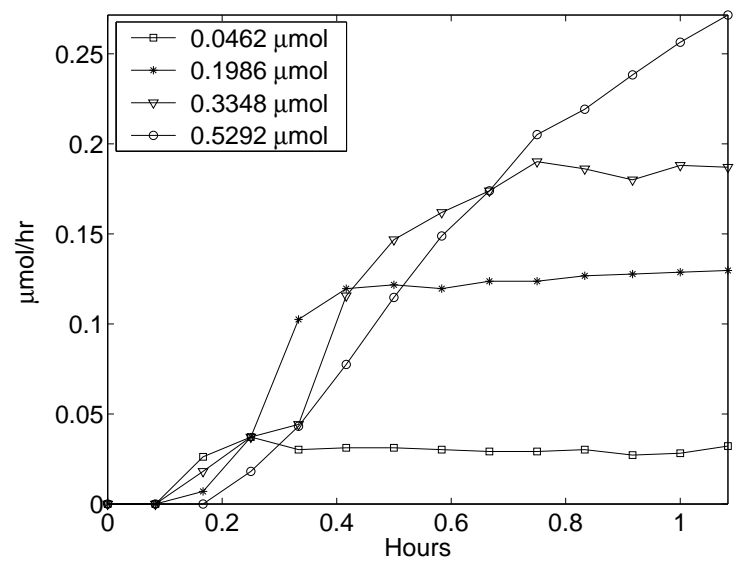

B

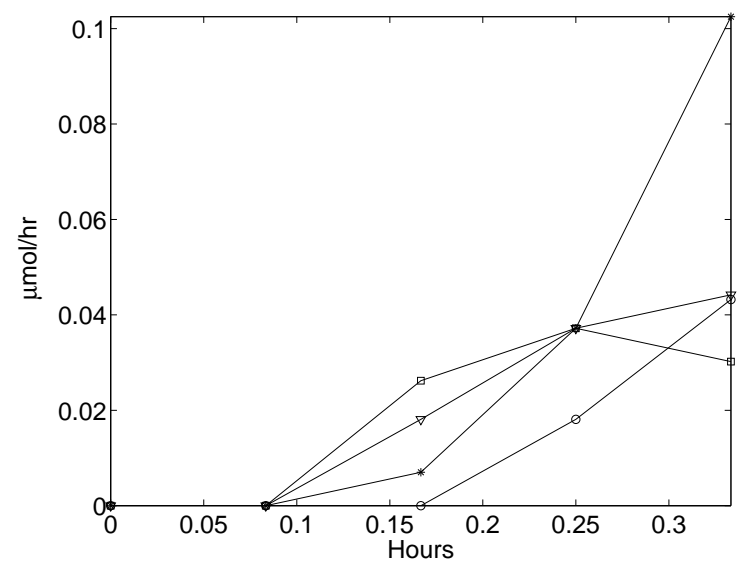

FIG. 2.1. Dose- (or concentration-) dependent lag evident in genistein biliary excretion rate [20]. Figure $1 B$ is a magnification of Figure 1A, focusing on the first 20 min of reported excretion.

$$
\begin{aligned}
& \frac{d C_{g e n}^{p p}}{d t}= \frac{Q_{p p} C_{g e n}^{B}-\frac{Q_{p p} C_{g e n}^{p p}}{P_{p p}}}{V_{p p}}, \\
& \frac{d C_{g e n}^{r p}}{d t}= \frac{Q_{r p} C_{g e n}^{B}-\frac{Q_{r p} C_{g e n}^{r p}}{P_{r p}}}{V_{r p}}-k_{u r i n e}^{g e n} C_{g e n}^{r p}, \\
& \frac{d C_{g e n}^{l}}{d t}= \frac{Q_{l} C_{g e n}^{B}-\frac{\left(Q_{l}+Q_{G I}\right) C_{g e n}^{l}}{P_{l}}+v_{i n f}(t)+\frac{Q_{G I} C_{g e n}^{G I}}{P_{r p}}}{V_{l}}-\frac{V_{m a x} C_{g e n}^{l}}{k_{m}+C_{g e n}^{l}} \\
& \frac{d C_{g e n}^{G I}}{d t}= \frac{Q_{G I} C_{g e n}^{B}-\frac{Q_{G I} C_{g e n}^{G I}}{P_{r p}}}{V_{G I}}, \\
& \frac{d C_{c o n}^{l}}{d t}= \frac{V_{m a x} C_{g e n}^{l}}{k_{m}+C_{g e n}^{l}}-k_{b i l e} C_{c o n}^{l}-\frac{\left(Q_{l}+Q_{G I}\right)\left(C_{c o n}^{l}-C_{c o n}^{R O B}\right)}{V_{l}} \\
& \frac{d C_{c o n}^{R O B}}{d t}= \frac{\left(Q_{l}+Q_{G I}\right)\left(C_{c o n}^{l}-C_{c o n}^{R O B}\right)}{V_{R O B}}-k_{u r i n e}^{c o n} C_{c o n}^{R O B}, \\
& \frac{d A_{c o n}^{b}}{d t}= k_{b i l e} C_{c o n}^{l}\left(t-d\left(t, C_{g e n}^{l}\right)\right) V_{l}, \\
& C_{g e n}^{B}=\frac{Q_{r p} C_{g e n}^{r p}}{P_{r p}}+\frac{Q_{p p} C_{g e n}^{p p}}{P_{p p}}+\frac{\left(Q_{l}+Q_{G I}\right) C_{g e n}^{l}}{P_{l}} \\
& Q_{t}
\end{aligned}
$$




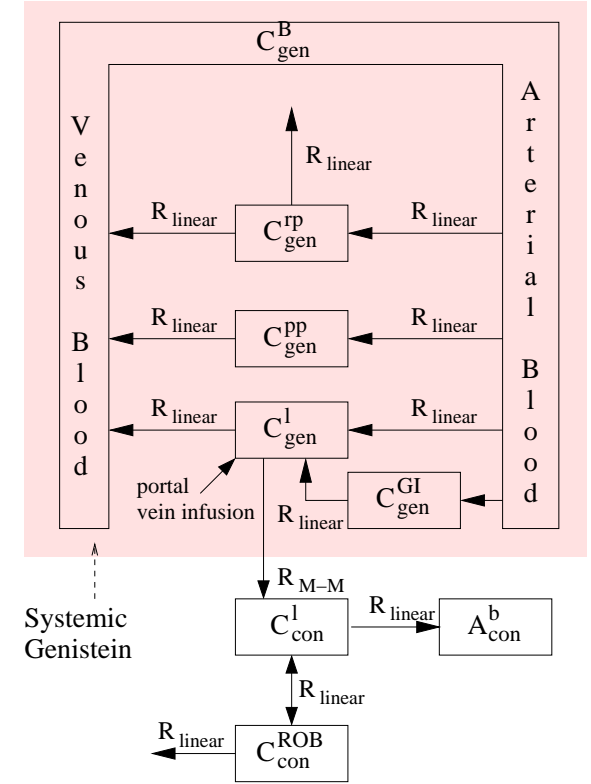

FIG. 2.2. Genistein PBPK model with a state-dependent delay in the biliary excretion compartment (see Table 6.1 for descriptions of abbreviations).

where the abbreviations used for the rates, volumes, and other terms are defined in Table 6.2.

We now describe the formulation of the venous infusion function $v_{\text {inf }}(t)$. When a chemical is infused into the portal vein, there is obviously a small but nonzero amount of time between the beginning of the infusion and the time at which the chemical reaches the liver at full dose, due to dispersion of the chemical in the bloodstream. A similar result occurs at the end of the infusion. Therefore we formulated the continuous function describing the venous infusions, $v_{\text {inf }}(t)$, by using a piecewisedefined cubic spline to capture the basic dynamics of this type of infusion. The formulation is as follows. A constant term $c_{v}$ is multiplied to the spline function, where $c_{v}$ is the constant venous infusion rate of GEN. The spline is defined as:

$$
v_{\text {inf }}(t)=\left\{\begin{array}{cc}
c_{v} F(t) & 0 \leq t<\epsilon \\
c_{v} G(t) & \epsilon \leq t \leq 1-\epsilon \\
c_{v} H(t) & 1-\epsilon<t \leq 1 \\
c_{v} I(t) & 1<t \leq 1+\frac{5}{60}
\end{array}\right.
$$

where we define

$$
F(t)=\frac{-2}{\epsilon^{3}} t^{3}+\frac{3}{\epsilon^{2}} t^{2},
$$

noting that $F(0)=0, \dot{F}(0)=\dot{F}(\epsilon)=0$, and $F(\epsilon)=1$,

$$
G(t)=1,
$$




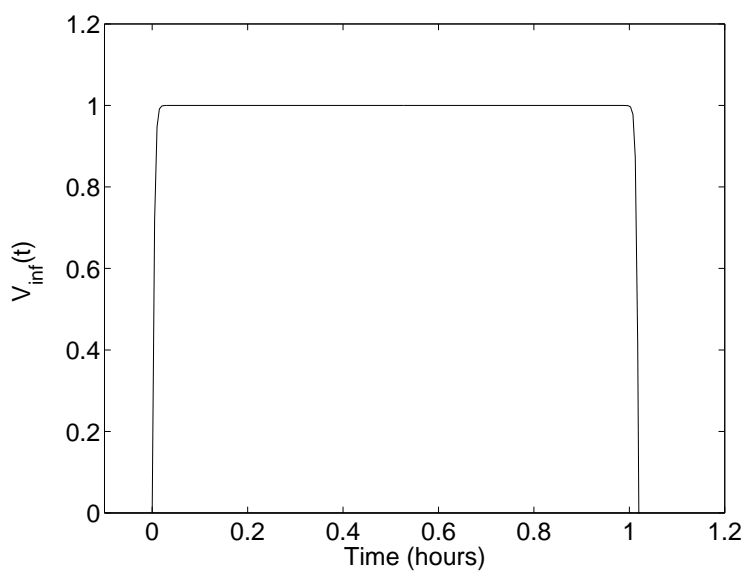

FIG. 2.3. Venous infusion function $V_{\text {inf }}(t)$ with $c_{v}=1$ and $\epsilon=0.05$. These values are used here for demonstration purposes and are not necessarily used in the model.

clearly with $G(\epsilon)=G(1-\epsilon)=1$ and $\dot{G}(\epsilon)=\dot{G}(1-\epsilon)=0$,

$$
H(t)=\frac{2}{\epsilon^{3}} t^{3}+\frac{3}{\epsilon^{3}}(\epsilon-2) t^{2}-\frac{6}{\epsilon^{3}}(\epsilon-1) t+\frac{1}{\epsilon^{3}}(3 \epsilon-2),
$$

such that $H(1-\epsilon)=1, \dot{H}(1-\epsilon)=\dot{H}(1)=0$, and $H(1)=0$,

$$
I(t)=0,
$$

obviously with all values of $I$ and $\dot{I}$ equal to zero. The value of $\epsilon$ is chosen to accurately represent a typical venous infusion under the experimental conditions outlined in [20]. The function is seen graphically in Figure 2.3.

We now formulate the delay function $d\left(t, C_{g e n}^{l}\right)$, which is given by

$$
d\left(t, C_{g e n}^{l}\right)=t_{\text {basal }}+c_{g e n} C_{g e n}^{l}(t) e^{-\frac{t}{c_{t}}} .
$$

By the nature of a bile duct cannulation, there is inevitably a delay in time from the beginning of biliary excretion of a chemical to the point where the bile has passed through the complex array of bile duct canaliculi, the main bile duct, down the bile duct catheter, and finally into the collection cannula. This delay is labeled $t_{\text {basal }}$. Added to that constant delay is a genistein-specific constant $c_{g e n}$, multiplied by the concentration of pure genistein $\left(C_{g e n}^{l}(t)\right)$ and a time-decaying term $e^{-\frac{t}{c_{t}}}$. This last term is added because the Sfakianos data suggest that the presence of the delay seems to become less apparent after a certain amount of time has elapsed during the infusions, as seen in Figure 2.1. These considerations are taken into account in the formulation of the state-dependent delay function (2.2). The parameters $t_{b a s a l}, c_{g e n}$, and $c_{t}$ are unknown and must be estimated. The values for these parameters are listed in Table 6.2 as "estimated" and are provided in Section 4.

Finally, the algebraic expression for the blood compartment $C_{\text {gen }}^{B}$ is obtained by assuming a pseudo steady-state for the differential equation describing that compartment. Setting the equation equal to zero allows for an explicit solution for $C_{g e n}^{B}$. This 
practice is standard in PBPK modeling and has the advantage of reducing the dimension of the system of equations by one. Computationally, this means our system of equations (2.1) can be numerically solved more efficiently.

3. Existence and Uniqueness. In this section we prove local existence and uniqueness of a solution to our nonlinear state-dependent DDE based on the work of Driver [25]. In his article, Driver presents a theorem for local existence and uniqueness of solutions to DDE of the type

$$
\dot{y}_{i}(t)=f_{i}\left(t, \vec{y}(t), \vec{y}\left(g_{2}(t, \vec{y}(t))\right), \cdots, \vec{y}\left(g_{m}(t, \vec{y}(t))\right)\right),
$$

for $i=1, \cdots, n$, and each $g_{j}(t, \vec{y}(t)) \leq t, j=2, \cdots, m$.

If we define $g_{1}(t, \vec{y}(t)) \equiv t$, the above equation (3) can be written in vector form as follows:

$$
\dot{\vec{y}}(t)=\vec{f}(t, \vec{y}(\vec{g}(t, \vec{y}(t))))
$$

where

$$
\vec{y}(\vec{g}(t, \vec{y}(t)))=\left(\vec{y}\left(g_{1}(t, \vec{y}(t))\right), \vec{y}\left(g_{2}(t, \vec{y}(t))\right), \cdots, \vec{y}\left(g_{m}(t, \vec{y}(t))\right)\right) .
$$

For the following development, we now introduce additional notation. We begin by defining

$$
(t, Y)=\left(t, y_{11}, \cdots, y_{1 n}, y_{21}, \cdots, y_{2 n}, \cdots, y_{m 1}, \cdots, y_{m n}\right)
$$

to be a vector in $\mathbb{R}^{m n+1}$. Let $(t, \vec{y})=\left(t, y_{11}, \cdots, y_{1 n}\right)$ be the projection of $(t, Y)$ into $\mathbb{R}^{n+1}$, the space of the first $n+1$ coordinates of $\mathbb{R}^{m n+1}$. Define

$$
f(t, Y)=\left(f_{1}(t, Y), \cdots, f_{n}(t, Y)\right)
$$

to be a real, $n$-dimensional, vector valued function over a domain $D \subset \mathbb{R}^{m n+1}$, where $D$ is open and connected. Let

$$
\vec{g}(t, \vec{y})=\left(g_{1}(t, \vec{y}), \cdots, g_{m}(t, \vec{y})\right)
$$

with $g_{1}(t, \vec{y})=t$ be a real, $m$-dimensional, vector-valued function over domain $D^{*}$, the projection of $D$ into $\mathbb{R}^{n+1}$. For a vector $v=\left(v_{1}, \cdots, v_{n}\right) \in \mathbb{R}^{n}$, we define the norm $\|v\|$ by

$$
\|v\|=\max _{1 \leq i \leq n}\left|v_{i}\right| .
$$

For a vector-valued function $\vec{h}(t, \vec{z})$ let the notation $\vec{h}(t, \vec{z}) \in(C$, Lip $)$ mean that throughout a region $R, \vec{h}(t, \vec{z})$ is continuous with respect to all variables and satisfies the Lipschitz condition $\|\vec{h}(t, \vec{z})-\vec{h}(t, \overrightarrow{\hat{z}})\| \leq A\|\vec{z}-\overrightarrow{\hat{z}}\|$ whenever $(t, \vec{z}),(t, \overrightarrow{\hat{z}}) \in R$, where $A$ is the Lipschitz constant.

Using these definitions, we now pose the following problem: 
Problem P: Let $f(t, Y)$ be defined over the domain $D$ and let $\vec{g}(t, \vec{y})$ be defined over $D^{*}$, with $g_{1}(t, \vec{y}) \equiv t$. For a fixed $t_{0} \in \mathbb{R}$, let $\alpha \in\left\{-\infty, t_{0}\right]$ be some fixed constant. Let $\phi(t)=\left(\phi_{1}(t), \cdots, \phi_{n}(t)\right)$ be a real, $n$-dimensional, vector-valued function of $t$ for $\alpha \leq t \leq t_{0}$. Note in the case that $\alpha=-\infty$ change all expressions of the form $\alpha \leq \cdots$, here and throughout this section, into $\alpha<\cdots$. Now, assuming that

$$
\begin{array}{ll}
\text { (i) } & \left(t_{0}, \phi\left(t_{0}\right)\right) \in D^{*} \\
\text { (ii }) & \alpha \leq g_{j}\left(t_{0}, \phi\left(t_{0}\right)\right) \leq t_{0} \text { for } j=2, \cdots, m, \text { and } \\
\text { (iii) } & \left(t_{0}, \phi\left(g\left(t_{0}, \phi\left(t_{0}\right)\right)\right)\right) \in D,
\end{array}
$$

we seek a real, $n$-dimensional, vector-valued function $\vec{y}(t)=\left(y_{1}(t), \cdots, y_{n}(t)\right)$, defined for $\alpha \leq t<\beta \leq \infty$, such that
(a) $\quad \vec{y}(t)=\phi(t)$
for $\alpha \leq t \leq t_{0}$
(b) $\vec{y}(t)$ is continuous
for $t_{0} \leq t<\beta$
(c) $(t, \vec{y}(t)) \in D^{*}$
for $t_{0} \leq t<\beta$
(d) $\quad \alpha \leq g_{j}(t, \vec{y}(t)) \leq t$
(e) $\quad(t, y(g(t, \vec{y}(t)))) \in D$
(f) $\dot{\vec{y}}(t)=\vec{f}(t, \vec{y}(g(t, \vec{y}(t))))$
for $t_{0} \leq t<\beta, j=1, \cdots, m$
for $t_{0} \leq t<\beta$, and
for $t_{0} \leq t<\beta$.

The following local existence and uniqueness result was proven in [25]:

THEOREM 3.1. Let $f(t, Y) \in(C$, Lip $)$ in each compact subset of $D$, let $\vec{g}(t, \vec{y}) \in$ $(C$, Lip $)$ in each compact subset of $D^{*}$, let $\alpha \leq g_{j}(t, \vec{y}) \leq t$ for all $(t, \vec{y}) \in D^{*} \cap\{t \geq$ $\left.t_{0}\right\}(j=2, \cdots, m)$, and let $\phi(t)$ be Lipschitz continuous on each finite subinterval of $\left[\alpha, t_{0}\right]$. Then there is a number $h>0$ such that a unique solution of $P$ exists for $\alpha \leq t<t_{0}+h$.

To apply the above local existence and uniqueness result to our problem, we state several assumptions and observations about our model and introduce appropriate notation for our model equations, variables, and functions to correspond to the notation used above.

Since all our state variables represent concentrations or amounts of genistein or its conjugates in specific tissues or bile, we assume every state variable is bounded below by zero. Since the rat does not produce genistein, we assume every state variable representing a concentration is bounded above by the total amount of genistein infused in the rat divided by the volume of the particular compartment for which the variable represents. Similarly, every compartment representing an amount is assumed to be bounded above by the total amount of genistein infused. Hence all of our state variables are bounded above. Furthermore, our time domain is bounded above by $T_{f}=1+5 / 60 h r$.

For our model, the initial condition is given by $\vec{\phi}(t)=\overrightarrow{0}$ where $\phi: D_{\phi} \rightarrow \mathbb{R}^{7}$ with $D_{\phi}=(-\infty, 0]$.

Defining $Y=\left(y_{11}, \cdots, y_{17}, y_{21}, \cdots, y_{27}\right)$, a vector in $\mathbb{R}^{14}$, and $\vec{y}=\left(y_{11}, \cdots, y_{17}\right)$, a vector in $\mathbb{R}^{7}$. That is, $y_{11}, \cdots, y_{17}$ correspond to the 7 state variables of our model and $y_{21}, \cdots, y_{27}$ correspond to the 7 state variables delayed by an amount of time admitted by the function $d\left(t, C_{g e n}^{l}\right)$ defined in Section 2 .

Now let $\vec{g}: D_{g} \rightarrow \mathbb{R} \times \mathbb{R}$ be defined by $\vec{g}(t, \vec{y})=\left(g_{1}(t, \vec{y}), g_{2}(t, \vec{y})\right)$, where $g_{1}(t, \vec{y})=$ $t, g_{2}(t, \vec{y})=t-d\left(t, C_{g e n}^{l}\right)$, and the domain $D_{g}$ is defined as follows:

$$
D_{g}=\left(-\infty, T_{f}\right] \times\left[0, M_{1}\right] \times \cdots \times\left[0, M_{7}\right]
$$


where $M_{1}, \ldots, M_{7}$ are defined to be our bounds on the state variables, as discussed above.

If we define the following variables

$$
\begin{array}{cl}
y_{11}=C_{g e n}^{p p}\left(g_{1}(t, \vec{y})\right) & y_{21}=C_{g e n}^{p p}\left(g_{2}(t, \vec{y})\right) \\
y_{12}=C_{g e n}^{r p}\left(g_{1}(t, \vec{y})\right) & y_{22}=C_{g g n}^{r p}\left(g_{2}(t, \vec{y})\right) \\
y_{13}=C_{g e n}^{l}\left(g_{1}(t, \vec{y})\right) & y_{23}=C_{g e n}^{l}\left(g_{2}(t, \vec{y})\right) \\
y_{14}=C_{g e n}^{G I}\left(g_{1}(t, \vec{y})\right) & y_{24}=C_{g e n}^{G I}\left(g_{2}(t, \vec{y})\right) \\
y_{15}=C_{\text {con }}^{l}\left(g_{1}(t, \vec{y})\right) & y_{25}=C_{c o n}^{l}\left(g_{2}(t, \vec{y})\right) \\
y_{16}=C_{c o n}^{R O B}\left(g_{1}(t, \vec{y})\right) & y_{26}=C_{c o n}^{R O B}\left(g_{2}(t, \vec{y})\right) \\
y_{17}=A_{\text {con }}^{b}\left(g_{1}(t, \vec{y})\right) & y_{27}=A_{c o n}^{b}\left(g_{2}(t, \vec{y})\right)
\end{array}
$$

then the system (2.1) takes the form $\dot{\vec{y}}=\vec{f}(t, Y)$, where

$$
\vec{f}(t, Y)=\left(f_{1}(t, Y), \cdots, f_{7}(t, Y)\right)
$$

Note that $\vec{f}(t, Y)$ is a real, 7-dimensional, vector-valued function over an open, connected subset $D$ of $\mathbb{R} \times \mathbb{R}^{14}$ defined as follows:

$$
\begin{gathered}
D=\left(-\infty, T_{f}\right] \times\left[0, M_{1}\right] \times \cdots \times\left[0, M_{7}\right] \\
\times\left[0, M_{1}\right] \times \cdots \times\left[0, M_{7}\right]
\end{gathered}
$$

We now state some trivial facts about our functions $\vec{f}(t, Y), \vec{g}(t, \vec{y})$ and $\vec{\phi}(t)$ that are needed to use Theorem 1 . Note that we will drop the vector notation in the expressions for these functions from this point on. Also note the following:

$$
\begin{gathered}
(0, \phi(0)) \in D_{g}, \\
-\infty<g_{2}(0, \phi(0)) \leq 0, \\
\left(0, \phi\left(g_{1}(0, \phi(0))\right), \phi\left(g_{2}(0, \phi(0))\right)\right) \in D .
\end{gathered}
$$

Also, since $\phi(t)=0$ for $t \in D_{\phi}$, it follows that $\phi(t)$ is Lipschitz continuous on each finite subinterval of $D_{\phi}$. Furthermore, we have the following:

Lemma 3.2. $-\infty<g_{2}(t, \vec{y}) \leq t$ for all $(t, \vec{y}) \in D_{g}$ such that $t \geq 0$.

Proof. $g_{2}(t, \vec{y})=t-\left(a+b y_{15} e^{-\frac{t}{c}}\right)$, for positive constants $a, b$, and $c$. Clearly $t-\left(a+b y_{15} e^{\frac{-t}{c}}\right) \leq t$ for all $t \geq 0$, since $y_{15} \geq 0$.

Lemma 3.3. $\vec{g}(t, \vec{y}) \in(C$, Lip $)$ in each compact subset of $D_{g}$.

Proof. Clearly if we show that both $g_{1}$ and $g_{2}$ are in $(C, L i p)$ in every compact subset of $D_{g}$, then $g \in(C, L i p)$ in every compact subset of $D_{g}$. Note $g_{1}(t, \vec{y})=t$, being the identity function, clearly has the desired properties.

Now, since the functions $t \mapsto t$ and $t \mapsto e^{-\frac{t}{c}}$ are obviously continuous on all compact sets, we have that $g_{2}(t, \vec{y})=t-\left(a+b y_{15} e^{-\frac{t}{c}}\right)$ is continuous in $t$ on all compact sets.

Let $D^{\prime}$ be a compact subset of $D_{g}$. Choose $(t, \vec{y}),(t, \overrightarrow{\hat{y}}) \in D^{\prime}$. Then $\| g_{2}(t, \vec{y})-$ $g_{2}(t, \overrightarrow{\hat{y}}) \|=$

$$
\left\|\left(t-\left(a+b y_{15} e^{-\frac{t}{c}}\right)\right)-\left(t-\left(a+b \hat{y}_{15} e^{-\frac{t}{c}}\right)\right)\right\|=\left\|b e^{-\frac{t}{c}}\left(\hat{y}_{15}-y_{15}\right)\right\|
$$


and since $b e^{-\frac{t}{c}}>0$ for all $t$,

$$
\left\|g_{2}(t, \vec{y})-g_{2}(t, \overrightarrow{\hat{y}})\right\|=b e^{-\frac{t}{c}}|| \hat{y}_{15}-y_{15}\left\|\leq b e^{0}|| \hat{y}_{15}-y_{15}\right\|=b|| \hat{y}_{15}-y_{15} \|
$$

Hence $g_{2}$ is Lipschitz in $\vec{y}$, and so it follows that $g$ is Lipschitz in $\vec{y}$. Therefore $\vec{g}(t, \vec{y}) \in(C$, Lip $)$ in each compact subset of $D_{g}$.

Lemma 3.4. $f(t, Y) \in(C$, Lip $)$ in each compact subset of $D$.

Proof. By regrouping and renaming constants, it can be shown that $f(t, Y)$ can be rewritten as $f(t, Y)=\left(f_{1}(t, Y), \cdots, f_{7}(t, Y)\right)^{T}$, where

$$
\begin{aligned}
f_{1}(t, Y)= & c_{1}^{f_{1}}\left(k_{1} y_{11}+k_{2} y_{12}+k_{3} y_{13}\right)+c_{2}^{f_{1}} y_{11} \\
f_{2}(t, Y)= & c_{1}^{f_{2}}\left(k_{1} y_{11}+k_{2} y_{12}+k_{3} y_{13}\right)+c_{2}^{f_{2}} y_{12} \\
f_{3}(t, Y)= & c_{1}^{f_{3}}\left(k_{1} y_{11}+k_{2} y_{12}+k_{3} y_{13}\right)+c_{2}^{f_{3}} y_{13} \\
& +v_{i n f}(t)+c_{3}^{f_{3}} y_{14}+\frac{c_{5}^{f_{3}} y_{13}}{c_{6}^{f_{3}}+y_{13}} \\
f_{4}(t, Y)= & c_{1}^{f_{4}}\left(k_{1} y_{11}+k_{2} y_{12}+k_{3} y_{13}\right)+c_{2}^{f_{4}} y_{14} \\
f_{5}(t, Y)= & \frac{c_{5}^{f_{3}} y_{13}}{c_{6}^{f_{3}}+y_{13}}+c_{1}^{f_{5}} y_{15}+c_{2}^{f_{5}}\left(y_{15}-y_{16}\right) \\
f_{6}(t, Y)= & c_{1}^{f_{6}} y_{16}+c_{2}^{f_{6}}\left(y_{15}-y_{16}\right) \\
f_{7}(t, Y)= & c_{1}^{f_{7}} y_{25}
\end{aligned}
$$

We will first show that $f$ is continuous in $t$ on every compact subset of $\left\{-\infty<t \leq T_{f}\right\}$. Note that the only explicit dependence of $t$ in (5) lies in the function $v_{\text {inf }}(t)=$ $c_{v} \tanh (\delta t)$. But $\tanh (\delta t)$ is continuous everywhere on the real line. Therefore it is continuous on every compact subset of $\left\{-\infty<t \leq T_{f}\right\}$, and hence so is $f$.

We now show $f$ is Lipschitz continuous on each compact subset of $D$ in each state variable. Note that if we show each $f_{i}$ is Lipschitz continuous on all compact subsets of $D$, then our assertion follows. So let $S$ be a compact subset of $D$, and choose $(t, Y),(t, \hat{Y}) \in S$.

We first consider $f_{3}$. Note that

$$
\begin{gathered}
\left\|f_{3}(t, Y)-f_{3}(t, \hat{Y})\right\|= \\
\|\left(c_{1}^{f_{3}}\left(k_{1} y_{11}+k_{2} y_{12}+k_{3} y_{13}\right)+c_{2}^{f_{3}} y_{13}+v_{i n f}(t)+c_{3}^{f_{3}} y_{14}+\frac{c_{5}^{f_{3}} y_{13}}{c_{6}^{f_{3}}+y_{13}}\right) \\
-\left(c_{1}^{f_{3}}\left(k_{1} \hat{y}_{11}+k_{2} \hat{y}_{12}+k_{3} \hat{y}_{13}\right)+c_{2}^{f_{3}} \hat{y}_{13}+v_{i n f}(t)+c_{3}^{f_{3}} \hat{y}_{14}+\frac{c_{5}^{f_{3}} \hat{y}_{13}}{c_{6}^{f_{3}}+\hat{y}_{13}}\right) \| \\
=\| c_{1}^{f_{3}} k_{1}\left(y_{11}-\hat{y}_{11}\right)+c_{1}^{f_{3}} k_{2}\left(y_{12}-\hat{y}_{12}\right)+\left(c_{1}^{f_{3}} k_{3}+c_{2}^{f_{3}}\right)\left(y_{13}-\hat{y}_{13}\right) \\
+c_{5}^{f_{3}}\left(\frac{y_{13}}{c_{6}^{f_{3}}+y_{13}}-\frac{\hat{y}_{13}}{c_{6}^{f_{3}}+\hat{y}_{13}}\right) \| \\
\leq c_{1}^{f_{3}} k_{1}\left\|y_{11}-\hat{y}_{11}\right\|+c_{1}^{f_{3}} k_{2}\left\|y_{12}-\hat{y}_{12}\right\|+\left(c_{1}^{f_{3}} k_{3}+c_{2}^{f_{3}}\right)\left\|y_{13}-\hat{y}_{13}\right\| \\
+c_{5}^{f_{3}}\left\|\frac{y_{13}}{c_{6}^{3}+y_{13}}-\frac{\hat{y}_{13}}{c_{6}^{f_{3}}+\hat{y}_{13}}\right\|
\end{gathered}
$$


Now note that

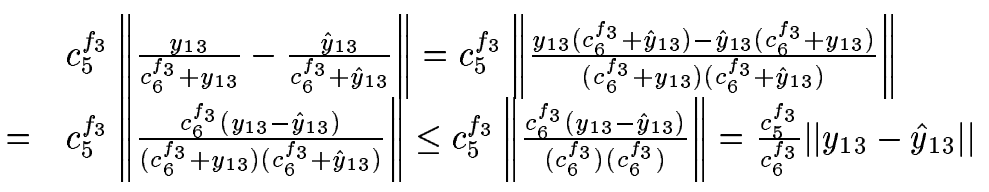

with $c_{5}^{f_{3}}, c_{6}^{f_{3}}>0$ and $y_{13}, \hat{y}_{13} \geq 0$.

Hence, it follows that

$$
\begin{gathered}
\left\|f_{3}(t, Y)-f_{3}(t, \hat{Y})\right\| \leq c_{1}^{f_{3}} k_{1}\left\|y_{11}-\hat{y}_{11}\right\|+c_{1}^{f_{3}} k_{2}\left\|y_{12}-\hat{y}_{12}\right\| \\
+\left(c_{1}^{f_{3}} k_{3}+c_{2}^{f_{3}}\right)\left\|y_{13}-\hat{y}_{13}\right\|+\frac{c_{5}^{f_{3}}}{c_{6}^{f_{3}}}\left\|y_{13}-\hat{y}_{13}\right\| \\
\leq c_{1}^{f_{3}} k_{1}\|Y-\hat{Y}\|+c_{1}^{f_{3}} k_{2}\|Y-\hat{Y}\|+\left(c_{1}^{f_{3}} k_{3}+c_{2}^{f_{3}}\right)\|Y-\hat{Y}\|+\frac{c_{5}^{f_{3}}}{c_{6}^{f_{3}}}\|Y-\hat{Y}\| \\
=\left(c_{1}^{f_{3}} k_{1}+c_{1}^{f_{3}} k_{2}+c_{1}^{f_{3}} k_{3}+c_{2}^{f_{3}}+\frac{c_{5}^{f_{3}}}{c_{6}^{f_{3}}}\right)\|Y-\hat{Y}\|
\end{gathered}
$$

Let $A=c_{1}^{f_{3}} k_{1}+c_{1}^{f_{3}} k_{2}+c_{1}^{f_{3}} k_{3}+c_{2}^{f_{3}}+\frac{c_{5}^{f_{3}}}{c_{6}^{f_{3}}}$. Noting that all terms in $A$ are positive constants, we have $A>0$. Hence, we have

$$
\left\|f_{3}(t, Y)-f_{3}(t, \hat{Y})\right\| \leq A\|Y-\hat{Y}\|
$$

which proves that $f_{3}$ is Lipschitz continuous on every compact subset of $D$, in all of its state variables, with Lipschitz constant $A$. Noting that $f_{1}, f_{2}, f_{4}, f_{5}$ and $f_{6}$ are all similar (simpler) cases to $f_{3}$, it follows that $f_{1}$ through $f_{6}$ are all Lipschitz continuous on every compact subset of $D$ in all state variables.

Recall $f_{7}(t, Y)=c_{1}^{f_{7}} y_{25}$. It is trivial to show this function is Lipschitz continuous in $y_{25}$ on all compact subsets of $D$. Therefore we have shown that $f \in(C, L i p)$ in each compact subset of $D$.

Now, applying Theorem 1, we obtain local existence and uniqueness of a solution to equation (1).

4. Inverse Problem. PBPK models require numerous parameters such as flow, metabolism, and elimination rate constants. In general, not all these parameters can be obtained from the literature or directly by experimental means. Therefore the unknown parameters must be estimated via, for example, the formulation of an inverse problem, that is, one compares experimental data (in our case given by [20]) to model predictions via a cost function and adjusts the unknown parameters to achieve a minimal cost. The resulting "optimized" parameters yielding this minimal cost will be the values used in the model. Mathematically, the inverse problem is formulated as follows: let $\vec{q}=\left(q_{1}, \cdots, q_{n}\right)$, where $q_{1}, \cdots, q_{n}$ are our $n$ unknown parameters. Then the inverse problem is defined as

$$
q_{o p t}^{\overrightarrow{o p}}=\min _{\vec{q} \in S}(J(\vec{q}))
$$

where $S=\left\{\vec{q} \in \mathbb{R}^{n}: q_{k}>0, k=1,2, \cdots, n\right\}$ and $J$ is our cost function to be defined. 
We considered several cost functions for this inverse problem, including least squares (LS), weighted least squares (WLS), and the maximum likelihood function (MLF). We compared the performances of these cost functions by using the optimized parameter set resulting from one function to obtain the cost this parameter set produces using the other two functions. With this comparison, we observed that the MLF consistently produced a parameter set that resulted in the best cost (lowest in the case of LS and WLS and highest in the case of MLF). Therefore we decided to use it exclusively throughout the estimation process. The MLF is defined as follows:

$$
\begin{aligned}
& J(\vec{q})=-\frac{1}{2} \sum_{i=1}^{r}\left[n_{i}(\log (2 \pi)+1)\right. \\
& \left.+\gamma_{i} \sum_{j=1}^{n_{i}} \log \left(f_{i, j}(\vec{q})\right)+n_{i} \log \left(\frac{1}{n_{i}} \sum_{j=1}^{n_{i}} \frac{\left(z_{i, j}-f_{i, j}(\vec{q})\right)^{2}}{\left(f_{i, j}(\vec{q})\right)^{\gamma_{i}}}\right)\right]
\end{aligned}
$$

where $r$ is the number of different data types being used, i.e., biliary excretion rate, cumulative biliary excretion, urinary elimination rate, etc., $n_{i}$ is the number of data points used in each data type, $z_{i, j}$ is each data point, where $i$ corresponds to the data type and $j$ corresponds to the time at which the data point represents, $f_{i, j}(\vec{q})$ is the model prediction at the time of the data point $z_{i, j}$, and finally each $\gamma_{i}$ are the heteroschedasticity terms for each data type, which regulate how the cost for each data type is determined. As $\gamma_{i}$ approaches zero, the cost function describes an absolute error model. As $\gamma_{i}$ approaches two, the cost function describes a relative error model. Each $\gamma_{i}$ is bounded between zero and two and is treated as an unknown parameter to be optimized in the inverse problem. Hence $\left\{\gamma_{1}, \gamma_{2}, \cdots\right\} \in \vec{q}$. For a derivation of (4.1), we refer the reader to [27].

In our problem, we use two data types: the experimental data consisting of biliary excretion rate data and the cumulative biliary excretion data obtained by numerically integrating the excretion rate data. Although the two data sets are based on the same reported data, we observed that using two different types enhanced the performance of the MLF, yielding better data fits. So we have two heteroschedasticity terms, $\gamma_{1}$ and $\gamma_{2}$, for the rate data and cumulative data, respectively.

The Nelder-Mead simplex-based method was the main optimization algorithm implemented for parameter optimization. This method proved to be most efficient generally leading to the lowest cost. The specific code used is fminsearch (MATLAB $R 11$, The MathWorks, Inc.). The results of the parameter optimization are presented in Table 6.3.

Model predictions of the experimental data using the optimized parameters are presented in Figures 4.2 and 4.1. In attempting to numerically solve the DDE, we found a high degree of stiffness. Therefore we implemented a three-stage, implicit, Runge-Kutta method (RADAR5, N. Guglielmi and E. Hairer, 2000), an effective numerical method for solving stiff, delay differential equations.

In the cumulative biliary excretion depicted in Figure 5, the simulated excretion curves for the lower two doses (Figure 5A) fit the data well. Note the slight underestimation for the lower dose and an overestimation followed by an underestimation for the higher dose. In the case of the higher two doses (Figure 5B), a key characteristic of the data is a crossing of the two excretion curves. Although the simulations begin with an underestimation and progress into a slight overestimation, they also cross, capturing this nontrivial characteristic of the state-dependent delay observed in the data.

The biliary excretion rate (i.e., the derivative of the cumulative excretion) is depicted in Figure 4. The fits to these data are not as close as the cumulative excretion data. This is to be expected since taking a derivative tends to amplify error. Note, 
A

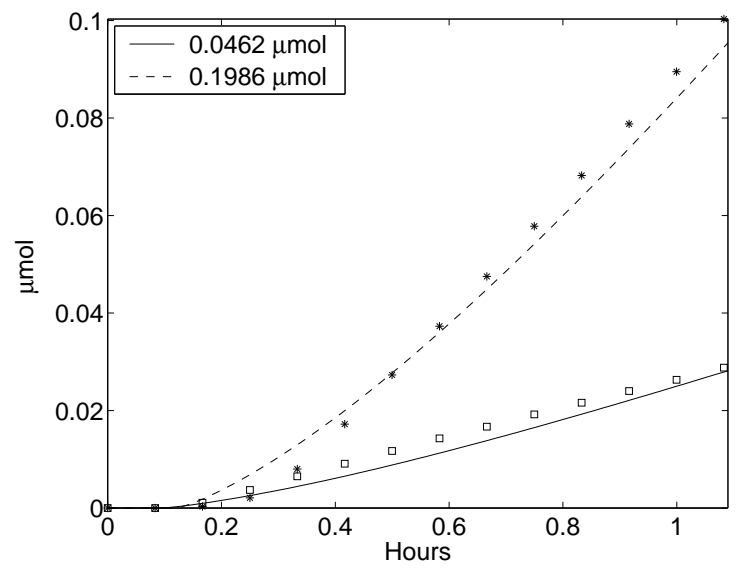

B

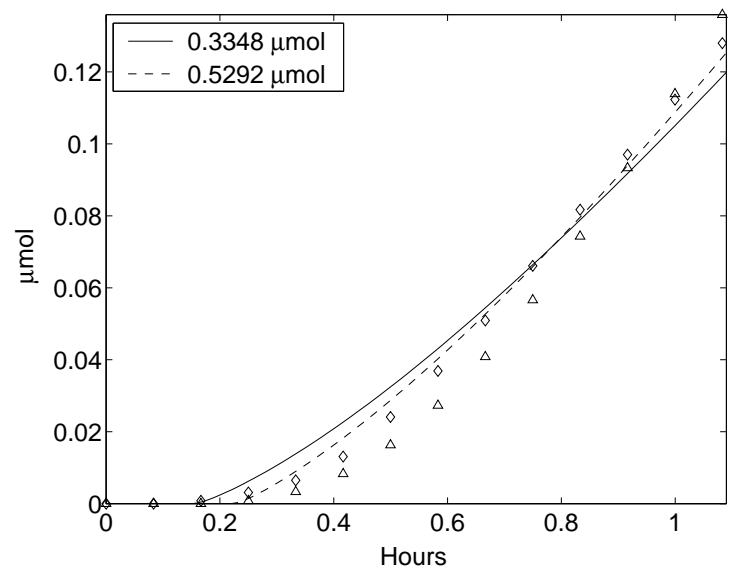

FIG. 4.1. Cumulative biliary excretion of genistein glucuronide after a 1 h venous infusion of pure genistein. Recall from Figure 1 that diamonds represent data from the lower dose in plot B.

however, that the fits to the data for the lower doses (Figure 4A) are better than for the upper doses. This possibly suggests a saturable process not accounted for in the model. However, the dynamics of the state dependence in the delay are more prominent in the derivative data, and the model decently characterizes these dynamics.

5. Statistical Significance of Delay Equations. In this section, we establish that our DDE model is a statistically significant improvement over the model based on standard ODE. To do this, we test the null hypothesis, that is, we compare the DDE model as described in the previous sections to the same model with the delay set to zero.

Let $\vec{q}$ be the vector of parameters in the DDE model to be optimized. Clearly, $\vec{q}$ is of dimension 10 (Table 6.3). Recall the delay function

$$
d\left(t, C_{g e n}^{l}\right)=t_{\text {basal }}+c_{g e n} C_{g e n}^{l}(t) e^{-\frac{t}{c_{t}}}
$$

with $t_{\text {basal }}, c_{g e n}$, and $c_{t}$ all elements of $\vec{q}$. To test the null hypothesis, we eliminate the delay function, thus eliminating three of the parameters in $\vec{q}$. We then optimize 


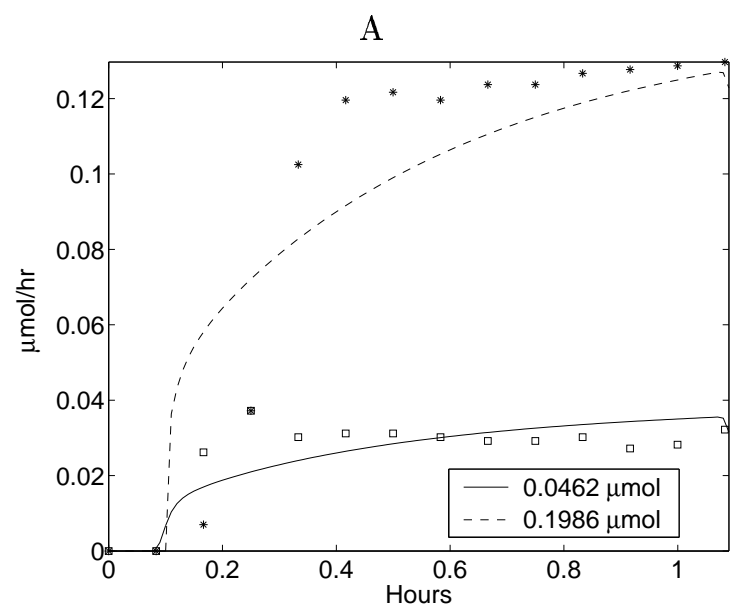

B

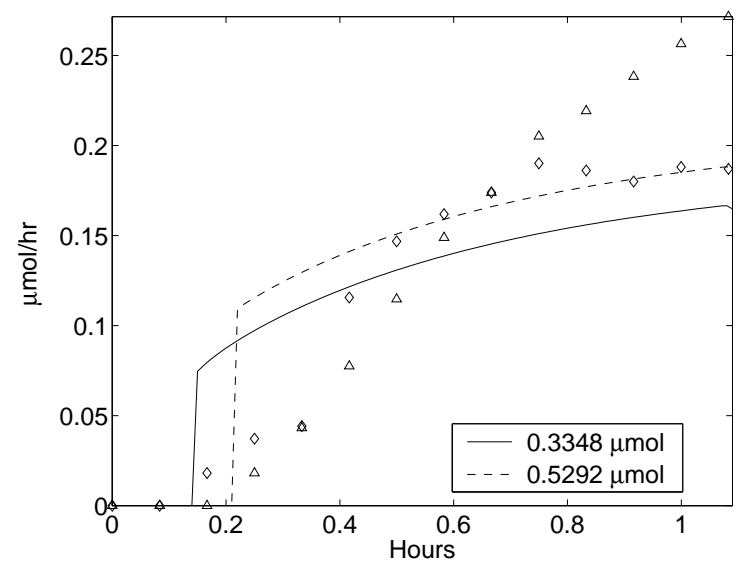

FIG. 4.2. Biliary excretion rate of genistein glucuronide after a 1 h venous infusion of pure genistein. Recall from Figure 1 that diamonds represent data from the lower dose in plot $B$.

over $\overrightarrow{\hat{q}}$, the vector of the remaining 7 unknown parameters, using a predetermined cost function (in this case, the MLF described in section 4). Then, using a test statistic $U$, we compare the optimal cost admitted by minimizer $\vec{q}$ to the optimal cost admitted by minimizer $\vec{q}$.

Using the developments in [28], we use the test statistic

$$
U=r\left|\frac{J(\overrightarrow{\widetilde{q}})-J(-\vec{q})}{J(-\vec{q})}\right|,
$$

where $r$ is the number of data points in the data set. After obtaining $U$, we choose a significance level $\alpha$ and use $\chi^{2}(s)$ tables to obtain the threshold $\tau$, where $s$ is the number of degrees of freedom. If we find that $U>\tau$, then we may conclude that the null hypothesis fails, i.e., the DDE model is a statistically significant improvement over the ODE model.

In our data set, $r=112$. We found $J(\vec{q})=597.2634$ and $J(\overrightarrow{\hat{q}})=402.0$. Recall that using the MLF, one wishes to maximize the likelihood. Hence, with the above 
TABLE 5.1

Resulting values from test statistic $U$.

\begin{tabular}{ccc}
\hline \multicolumn{3}{c}{$\chi^{2}(3)$} \\
\hline Confidence Level (\%) & $\alpha$ & Threshold Value $\tau$ \\
\hline $1 \%$ & 0.99 & 0.115 \\
$10 \%$ & 0.9 & 0.584 \\
$90 \%$ & 0.1 & 6.251 \\
$95 \%$ & 0.05 & 7.815 \\
$99 \%$ & 0.01 & 11.34 \\
\hline
\end{tabular}

numbers, $J(-\overrightarrow{\mathscr{q}})$ is "better" than $J(\overrightarrow{\widehat{q}})$. Statistical significance is seen by the following. From our above equation, we compute the test statistic $U=36.6162$. Noting that we have 3 degrees of freedom, we compute the thresholds for the stated confidence levels in Table 1. So we see that even with a $99 \%$ confidence level, $U>\tau$. Therefore the null hypothesis fails. This clearly shows a statistically significant improvement in simulating the data by implementing the DDE model.

6. Discussion. Though the delay observed in the data is present in the model simulations, the true dynamics of the delay are clearly not captured with great accuracy. The failure to accurately capture these dynamics limits the ability of the model to fit biliary excretion rate data. A possible solution is using a different numerical optimization method. Another possible solution is developing a high-fidelity model with more biological consideration for biliary excretion. Such a model may include a distributed parameter system for the liver compartment based on dispersion models that have been developed for chemical reactors. The advantage in using a distributed model is that it is able to account for concentration gradients within the bile duct and liver acinus, where a compartmental model, even with delays, cannot. We are currently developing such a model. Further experimental investigation combined with the modeling efforts described may ultimately be needed to fully understand the nature of the observed delay.

Since there are two urinary elimination rate constants in the model and no urinary elimination data to compare under the experimental conditions described in section 2, the optimized parameters obtained cannot be unique. As seen in Table 6.3 , the estimate for the rate constant governing the urinary elimination of genistein conjugates $\left(k_{\text {urine }}^{\text {con }}\right)$ is essentially zero. This is not suggested by experimental data [21]. However, if we bound $k_{u r i n e}^{c o n}$ away from zero, by adjusting the rate constant for urinary elimination of pure genistein, we can obtain similar data fits to what is seen in Figures 4.2 and 4.1. Hence, even local uniqueness of our parameter set is not possible. Therefore data for urinary excretion of genistein under similar experimental conditions are desired. Using those data to estimate the unknown kinetic parameters governing the urinary elimination of the parent compound, metabolites, or both will hopefully yield identifiability of the parameters. For a thorough discussion on identifiability and consistency of estimators we refer the interested reader to Banks and Kunisch [28].

The estimated parameters and system of DDE defining our model can be incorporated in a full PBPK model capable of predicting a wide range of genistein dosimetry under various experimental conditions. If the urinary elimination data mentioned above are obtained, we can use the full PBPK model to estimate the urinary elimina- 
tion parameters. Ultimately, this approach can serve as a template for other chemicals that undergo enterohepatic circulation. 


\section{Appendix}

TABLE 6.1

Abbreviations defined for PBPK model.

\begin{tabular}{ll}
\hline Abbreviation & Description \\
\hline$C_{g e n}^{B}$ & Concentration of pure genistein in plasma $(\mu \mathrm{mol} / \mathrm{hr})$ \\
$C_{g e n}^{r p}$ & Concentration of pure genistein in richly perfused tissues \\
$C_{g e n}^{\text {gp }}$ & Concentration of pure genistein in poorly perfused tissues \\
$C_{g e n}^{l}$ & Concentration of pure genistein in liver \\
$C_{g e n}^{G I}$ & Concentration of pure genistein in gastro-intestinal \\
$C_{\text {con }}^{l}$ & (GI) tract lumen \\
$C_{\text {con }}^{R O B}$ & Concentration of genistein conjugates in liver \\
$A_{\text {con }}^{b}$ & Concentration of genistein conjugates in rest of body (ROB) \\
& Amount of genistein conjugates in bile \\
$R_{\text {linear }}$ & - delayed compartment $(\mu m o l)$ \\
$R_{M-M}$ & Linear rate constant - see Figure $2.2\left(h r^{-1}\right)$ \\
\hline
\end{tabular}


TABLE 6.2

Abbreviations defined for model equations.

\begin{tabular}{|c|c|c|}
\hline Abbreviation & Description & Value \\
\hline$m_{\text {rat }}$ & Mass of rat (value from [20]) & $0.25(\mathrm{~kg})$ \\
\hline$Q_{p p}$ & $\begin{array}{l}\text { Blood flow rate through } \\
\text { poorly perfused tissue }\end{array}$ & $0.528 Q_{t}(L / h r)$ \\
\hline$Q_{r p}$ & $\begin{array}{l}\text { Blood flow rate through } \\
\text { richly perfused tissue }\end{array}$ & $0.289 Q_{t}(L / h r)$ \\
\hline$Q_{l}$ & $\begin{array}{l}\text { Blood flow rate through } \\
\text { liver }\end{array}$ & $0.03 Q_{t}(L / h r)$ \\
\hline$Q_{G I}$ & $\begin{array}{l}\text { Blood flow rate through } \\
\text { GI tract }\end{array}$ & $0.153 Q_{t}(L / h r)$ \\
\hline$Q_{t}$ & $\begin{array}{l}\text { Blood flow rate through } \\
\text { heart (cardiac output) }\end{array}$ & $14.1\left(m_{r a t}\right)^{0.75}(L / h r)$ \\
\hline$P_{p p}$ & $\begin{array}{l}\text { Blood/tissue partition } \\
\text { coefficient for poorly } \\
\text { perfused tissue }\end{array}$ & 0.59 \\
\hline$P_{r p}$ & $\begin{array}{l}\text { Blood/tissue partition } \\
\text { coefficient for richly } \\
\text { perfused tissue }\end{array}$ & 1.94 \\
\hline$P_{l}$ & $\begin{array}{l}\text { Blood/tissue partition } \\
\text { coefficient for liver }\end{array}$ & 3.61 \\
\hline$V_{p p}$ & Volume of poorly perfused tissue & $0.1519(L)$ \\
\hline$V_{r p}$ & Volume of richly perfused tissue & $0.0064(L)$ \\
\hline$V_{l}$ & Volume of liver & $0.0092(L)$ \\
\hline$V_{G I}$ & Volume of GI tract & $0.0065(L)$ \\
\hline$V_{R O B}$ & Volume of ROB & $0.2409(L)$ \\
\hline$k_{\text {urine }}^{\text {gen }}$ & $\begin{array}{l}\text { Linear rate term for pure genistein } \\
\text { excretion in urine }\end{array}$ & estimated $\left(h r^{-1}\right)$ \\
\hline$k_{\text {urine }}^{\text {con }}$ & $\begin{array}{l}\text { Linear rate term for genistein } \\
\text { conjugate excretion in urine }\end{array}$ & estimated $\left(h r^{-1}\right)$ \\
\hline$k_{\text {bile }}$ & $\begin{array}{l}\text { Linear rate term for genistein } \\
\text { conjugate excretion in bile }\end{array}$ & estimated $\left(h r^{-1}\right)$ \\
\hline$V_{\max }, k_{m}$ & $\begin{array}{l}\text { Michaelis-Menton rate terms for } \\
\text { saturable congugation of genistein } \\
\text { in liver }\end{array}$ & $\begin{array}{l}\text { estimated } \\
(\mu m o l / L / h r, \mu m o l / L)\end{array}$ \\
\hline$c_{v}$ & $\begin{array}{l}\text { Venous infusion rate of pure } \\
\text { genistein }\end{array}$ & varies (check Figure 2.1) \\
\hline$\delta$ & $\begin{array}{l}\text { Constant term used in adjusting } \\
\text { venous infusion function } v_{i n f}(t)\end{array}$ & 500 \\
\hline$t_{\text {basal }}$ & $\begin{array}{l}\text { Constant term used as a basal } \\
\text { biliary excretion delay in delay } \\
\text { function } d\left(t, C_{g e n}^{l}\right)\end{array}$ & estimated $(h r)$ \\
\hline$c_{g e n}$ & $\begin{array}{l}\text { Constant term used as a genistein- } \\
\text { specific delay in delay function } \\
d\left(t, C_{g e n}^{l}\right)\end{array}$ & $\begin{array}{l}\text { estimated } \\
(L \cdot h r / \mu m o l)\end{array}$ \\
\hline$c_{t}$ & $\begin{array}{l}\text { Constant term used in delay function } \\
d\left(t, C_{g e n}^{l}\right)\end{array}$ & estimated $(h r)$ \\
\hline
\end{tabular}


TABLE 6.3

Resulting values from parameter optimization.

\begin{tabular}{ll}
\hline Parameter & Value \\
\hline$k_{\text {urine }}^{\text {gen }}$ & $28.2 \mathrm{hr}^{-1}$ \\
$k_{\text {urine }}^{\text {con }}$ & $1 \times 10^{-34} \mathrm{hr}^{-1}$ \\
$k_{\text {bile }}$ & $111.7 \mathrm{hr}^{-1}$ \\
$V_{\text {max }}$ & $25.3 \mu \mathrm{mol} / \mathrm{L} / \mathrm{hr}$ \\
$k_{m}$ & $0.62 \mu \mathrm{mol} / \mathrm{L}$ \\
$t_{\text {basal }}$ & $0.078 \mathrm{hr}$ \\
$c_{\text {gen }}$ & $0.066 \mathrm{~L} \cdot \mathrm{hr} / \mu \mathrm{mol}$ \\
$c_{t}$ & $1.0 \mathrm{hr}$ \\
$\gamma_{1}$ & 2 \\
$\gamma_{2}$ & 2 \\
\hline
\end{tabular}

\section{REFERENCES}

[1] T. Colburn, F.S. vom SaAl, And A.M. Soto, Developmental Effects of Endocrine-Disrupting Chemicals in Wildlife and Humans, Environ. Health Perspect., 101 (1993), pp. 378-384.

[2] R. Stone, Science, 256 (1994), pp. 308-310.

[3] S.H. SAFE, Environmental and Dietary Estrogens and Human Health: Is There a Problem? , Environ. Health Perspect., 103 (1995) pp. 346-351.

[4] M. Messina, V. Persky, K.D.R. Setchell, And S. Barnes, Soy intake and cancer risk: a review of the in vitro and in vivo data, Nutr. Cancer 21 (1994), pp. 113-131.

[5] K.D.R. SETChell AND A. CASSIDY, Dietary isoflavones: biological effects and relevance to human health, J. Nutr. 129 (1999), pp. 758S-767S.

[6] K.D.R. Setchell, M.B. WelSh, AND C.K. LiM, High-performance liquid chromatographic analysis of phytoestrogens in soy protein preparations with ultraviolet, electrochemical and thermospray mass spectrometric detection, J. Chromatography 386 (1987), pp. 315-323.

[7] K.D.R. Setchell, L.Z. Nechemias-Zimmer, J. CaI, And J.E. Heubi, Exposure of infants to phyto-oestrogens from soy-based infant formula, Lancet 350 (1997), pp. 23-27.

[8] R. Hakkak, S. Korourian, M. Ronis, D. Irby, S. Kechelava, J. Benson, C. Weatherford, J. PARKER, AND T.M. BADGER, Dietary soy protein isolate or whey protein protects against AOM-induced colon cancer in male rats, Proc. Am. Assoc. Cancer Res. 40 (1999), pp. 56.

[9] C.A. Lamartiniere, J. Moore, M. Holland, and S. Barnes, Neonatal genistein chemoprevents mammary cancer, Proc. Soc. Exp. Biol. Med. 208 (1995), pp. 120-123.

[10] S. BARnes And T.G. Peterson, Biochemical targets of the isoflavone genistein in tumor cell lines, Proc. Soc. Exp. Biol. Med. 208 (1995), pp. 103-108.

[11] R.C. Santell, Y.C. Chang, M.G. NAIR, And W.G. Helferich, Dietary genistein exerts estrogenic effects upon the uterus, mammary gland and the hypothalamic/pituitary axis in rats, J. Nutr. 127 (1997) pp. 263-269.

[12] K.M. Flynn, S.A. Ferguson, K.B. Delclos, and R.R. Newbold, Effects of genistein exposure on sexually dimorphic behaviors in rats., Toxicol. Sci. 55 (2000), pp. 311-319.

[13] H.P. Lee, L. Gourley, S.W. Duffy, J. Esteve, J. Lee, And N.E. Day, Dietary effects on breast-cancer risk in Singapore, Lancet 336 (1991), pp. 1197-1200.

[14] A.H. Wu, R.G. Ziegler, P.L. Horn-Ross, M.Y. Nomura, D.W. West, L.N. Kolonel, J.F. Rosenthal, R.N. Hoover, AND M.C. PIKe, Tofu and risk of breast cancer in AsianAmericans, Cancer Epidemiol. Biomarkers Prev. 5 (1996), pp. 901-906.

[15] R.G.Ziegler, R.N. Hoover, R.N. Hildeshein, Et Al., Migration patterns and breast cancer risk in Asian-American women, J. Natl. Cancer Inst. 85 (1993) pp. 1819-1827.

[16] T.G. Peterson and S. Barnes, Mol. Biol. Cell. 5 (1994), pp. 384a.

[17] W.A. Fritz, L. Coward, J. WANG, ANd C.A. Lamartiniere, Dietary genistein: perinatal mammary cancer prevention, bioavailability and toxicity testing in the rat, Carcinogenesis 19 (1998), pp. 2151-2158.

[18] F.O. StePhens, Phytoestrogens and prostate cancer: possible preventive role, Med. J. Aust. 167 (1997), pp. 138-140. 
[19] J.R. Levy, K.A. Faber, L. Ayyash, And C.L. Hughes, JR., The effect of prenatal exposure to the phytoestrogen genistein on sexual differentiation in rats, Proc. Soc. Exp. Biol. Med. 208 (1995), pp. 60-66.

[20] J. Sfakianos, L. CowArd, M. Kirk, AND S. BARnes, Intestinal uptake and biliary excretion of the isoflavone genistein in rats, J. Nutr. 127 (1997) pp. 1260-1268.

[21] T. Yasuda, S. Mizunuma, Y. Kano, K. Saito, and K. Ohsawa, Urinary and biliary metabolites of genistein in rats, Biol. Pharm. Bull. 19 (1996), pp. 413-417.

[22] Federal Register: December 28, 1998 (Volume 63, Number 248), pp. 71541-71568.

[23] Physiological Parameter Values for PBPK Models, ILSI (International Life Sciences Institute), (1994).

[24] D.R. Plowchalk, Physiologically-Based Models for Estradiol Pharmacokinetics in Rats and Humans: Report prepared for the American Chemistry Counsel (formerly the Chemical Manufacturers Association) (1997).

[25] R.D. DrIVER, Existence Theory for a Delay-Differential System, Cont. Diff. Eqs. 1 (1963), pp. 317-336.

[26] P.M. Schlosser, M.G. ZaGer , And H.A. Barton, Final report for the American Chemistry Counsel (1999) (Not published, not for citation).

[27] C.E. Cole, H.T. Tran, AND P.M. SChlosser, Physiologically based pharmacokinetic modeling of benzene metabolism in mice through extrapolation from in vitro to in vivo, J. Tox. Env. Health A(62) (2001), pp. 439-465.

[28] H.T. BANKS AND K. Kunisch , Estimation Techniques for Distributed Parameter Systems, Birkhauser, Boston, MA, 1989.

[29] Y.H. Ju, C.D. Allred, K.F. Allred, K.L. Karko, D.R. Doerge, And W.G. Helferich, Physiological concentrations of dietary genistein dose-dependently stimulate growth of estrogen-dependent human breast cancer (MCF-7) tumors implanted in athymic nude mice, J. Nutr. 131 (2001) pp. 2957-2962.

[30] K.B. Delclos, T.J. Bucci, L.G. Lomax, J.R. Latendresse, A. Warbritton, C.C. Weis, AND R.R. NewBold, Effects of dietary genistein exposure during development on male and female CD (Sprague-Dawley) rats, Repro. Toxicol. 15 (2001) pp. 647-663.

[31] E.M. Laurenzana, C.C. Weis, C.W. Bryant, R. Newbold, and K.B. Delclos, Effect of dietary administration of genistein, nonylphenol or ethinyl estradiol on hepatic testosterone metabolism, cytochrome P-450 enzymes, and estrogen receptor alpha expression, Food Chem. Toxicol. 40 (2002), pp. 53-63.

[32] M.J. Messina AND C.L.LOPRINZI, Soy for breast cancer survivors: a critical review of the literature, J. Nutr. (2001), pp. 3095S-3108S.

[33] R.R. Newbold, E. Padilla-Banks, B. Bullock, and W.N. Jefferson, Uterine adenocarcinoma in mice treated neonatally with genistein, Cancer Res. 61 (2001), pp. 4325-4328. 